Robert W. Vallin, Mathematics Department, Slippery Rock University, 229 Vincent Science Hall, Slippery Rock, PA 16057-1326. e-mail:

robert.vallin@sru.edu

\title{
NEWHOUSE THICKNESS AND POROSITY OF CANTOR SETS
}

\begin{abstract}
In this paper we compare and contrast two indicators of size in the real line: porosity (porosity index) and thickness. We use a relationship between thickness and the porosities to find a condition on when the sum of two Cantor sets is an interval. Finally, some questions are posed.
\end{abstract}

\section{Introduction and Definitions}

There are several ideas in the literature concerning what makes a set in the real line small (or alternatively large). Of course not all of these definitions are equivalent. For example, sets that are measure zero needn't be nowhere dense. We will concentrate on two indicators of size: porosity and porosity index, which look at how small a Cantor set can be, and thickness, which tells how large a Cantor set can be. At first glance the definitions of thickness and porosity index make it seem like one could be the reciprocal of the other. However, this is not the case. In this paper we shall find conditions on a Cantor set under which the porosity/porosity index gives information about the thickness. We include an example to show these two ideas are not always related and give some questions to be answered.

We start with the definition of porosity for a set on the line. Let $E$ be a set in $\mathbb{R}$ and $x \in \mathbb{R}$. The right porosity of $E$ at $x, p^{+}(E, x)$ is given by

$$
p^{+}(E, x)=\limsup _{\varepsilon \rightarrow 0^{+}} \frac{k}{k+h}
$$

Key Words: thickness, porosity, Cantor set, sum of Cantor sets

Mathematical Reviews subject classification: 28A05

Received by the editors March 14, 2001 
where $(x+h, x+h+k) \cap E=\emptyset, h+k<\varepsilon$, and $h, k>0$. In a similar vein, the left porosity of $E$ at $x$ (written $p^{-}(E, x)$ ) is defined by having $(x-h-k, x-h) \cap E=\emptyset$. Finally, the porosity of $E$ at $x$ is defined by

$$
p(E, x)=\max \left\{p^{-}(E, x), p^{+}(E, x)\right\} .
$$

Values of $p$ lie in $[0,1]$. We say that $E$ is porous at $x$ if $p(E, x)>0$ and that $E$ is a porous set if $p(E, x)$ is positive for all $x$ in $E$. Stronger is the claim that $E$ is uniformly porous. A set $E$ is uniformly porous if there exists an $\alpha>0$ so that

$$
p(E)=\inf _{x \in E}\{p(E, x)\} \geq \alpha .
$$

For example, the Cantor ternary set $C$ is uniformly porous. We can easily see that $p(C) \geq 1 / 2$.

A closely related concept we refer to as the porosity index. Let $E$ be a set in $\mathbb{R}$ and $x \in \mathbb{R}$. The right porosity index of $E$ at $x, p i^{+}(E, x)$ is given by

$$
p i^{+}(E, x)=\limsup _{\varepsilon \rightarrow 0^{+}} \frac{k}{h}
$$

where $(x+h, x+h+k) \cap E=\emptyset, h+k<\varepsilon$, and $h, k>0$. The left porosity index of $E$ at $x$ (written $p i^{-}(E, x)$ ) is defined with $(x-h-k, x-h) \cap E=\emptyset$ and then the porosity index of $E$ at $x$ is defined by

$$
p i(E, x)=\max \left\{p i^{-}(E, x), p i^{+}(E, x)\right\} .
$$

For the porosity index, the values lie in $[0, \infty)$. We say $E$ is uniformly indexed if there exists an $\alpha>0$ such that

$$
i(E)=\inf _{x \in E}\{p i(E, x)\} \geq \alpha .
$$

Continuing our Cantor set example, $i(C) \geq 1$.

As would be expected, the concepts of porosity and porosity index are related to one another. This relation is given in the following lemma from the Appendix of [6].

Lemma 1. If $p=p^{+}(E, x)$ and $i=p i^{+}(E, x)$ then

$$
p=\frac{i}{1+i} \text { and } i=\frac{p}{1-p} .
$$

More on porosity and its applications may be found in [6] and [7].

We now turn our attention to Cantor sets. There are two points of view we will take. The first (although it may be generalized) is rather strict. We 
shall call this construction the standard construction of a Cantor set. Let $\left\{a_{n}\right\}$ be a sequence of values where $0<a_{n}<1 / 2$. Stage zero of the construction consists of the closed interval $I$. At stage $n$ we have $2^{n}$ closed intervals $I_{n, k}$, $k=1,2, \ldots, 2^{n}$ of length $\left(a_{1} \cdot a_{2} \cdots a_{n-1}\right)|I|$ where $|I|$ denotes the length of $I$. In the next step each sub-interval $I_{n, k}$ is divided into three pieces $I_{n, k}^{0}, O_{n, k}$, and $I_{n, k}^{1}$ where $O_{n, k}$ is an open interval between $I_{n, k}^{0}$ and $I_{n, k}^{1}, I_{n, k}^{0}$ has the same left endpoint as $I_{n, k}, I_{n, k}^{1}$ has the same right endpoint as $I_{n, k}$, and

$$
\frac{\left|I_{n, k}^{0}\right|}{\left|I_{n, k}\right|}=\frac{\left|I_{n, k}^{1}\right|}{\left|I_{n, k}\right|}=a_{n}
$$

The Cantor set is then

$$
\bigcap_{n=1}^{\infty}\left(\bigcup_{k=1}^{2^{n}} I_{n, k}\right)
$$

We will denote this set by $C_{a_{n}, a_{n}}$ (or $C_{a, a}$ if $\left\{a_{n}\right\}$ is the constant sequence $\{a\}$ ). Note the Cantor ternary set would be written as $C_{1 / 3,1 / 3}$. The terminology we will employ is to call $C_{a_{n}, a_{n}}$ a uniform Cantor set and $C_{a, a}$ a constant Cantor set.

For our second point of view, we will consider a Cantor set to be any set of the form

$$
I \backslash \cup_{i=1}^{\infty} O_{i}
$$

where $I$ is a bounded, closed interval and $\left\{O_{i}: i \geq 1\right\}$ is a countable collection of disjoint open intervals which have no endpoint in common and whose union is dense in $I$. We take the following approach to the construction of the Cantor set. Let $I$ be the zeroth level. Assume we have defined the construction up to the $n$th level. Let $I^{\sigma}$ (where $\sigma$ is a string of length $n$ consisting of 0 's and 1's which gives the address of the interval) be an $n$th level interval. Then define $O_{I^{\sigma}}$ as the interval of least index contained in $I^{\sigma}$ and let $I^{\sigma 0}$ and $I^{\sigma 1}$ be the closed intervals where

$$
I^{\sigma}=I^{\sigma 0} \cup O_{I^{\sigma}} \cup I^{\sigma 1} .
$$

Continue this process inductively and our Cantor set is given by

$$
C=\bigcap_{n=1}^{\infty}\left(\bigcup_{|\sigma|=n} I^{\sigma}\right)
$$

The end result is always $C$, but, if we look the set $\bigcap_{n=1}^{k}\left(\bigcup_{|\sigma|=n} I^{\sigma}\right)$, it is a different set for different ordering of $\left\{O_{i}: i \geq 1\right\}$. For a specific $\left\{O_{i}: i \geq 1\right\}$ we 
would refer to this construction of $C$ as the derivation (written $\mathcal{D}$ ) of $C$ with respect to $\left\{O_{i}: i \geq 1\right\}$. The different derivations help motivate the concept of thickness.

Definition 1. Given a derivation $\mathcal{D}, A \in \mathcal{D}$ refers to an arbitrary interval at some level $n$ which will be decomposed into $A^{0} \cup O_{A} \cup A^{1}$ where $O_{A}$ refers to the interval of least index which is contained in $A$. Then the thickness, $\tau$, of the Cantor set $C$ is defined by

$$
\tau(C)=\infty \text { if }\left\{O_{i}: i \geq 1\right\}=\emptyset
$$

otherwise

$$
\tau(C)=\sup _{\mathcal{D}}\left(\inf _{A \in \mathcal{D}}\left(\min \left\{\frac{\left|A^{0}\right|}{\left|O_{A}\right|}, \frac{\left|A^{1}\right|}{\left|O_{A}\right|}\right\}\right)\right) .
$$

For more on thickness consult [1], [2], [4], and [5].

Finding the thickness is reasonably easy if we know the derivation is ordered. We say a derivation is ordered if for intervals $A$ and $B$ in the derivation with $A=A^{0} \cup O_{A} \cup A^{1}, B=B^{0} \cup O_{B} \cup B^{1}$, and $B \subseteq A$, then $\left|O_{A}\right| \geq\left|O_{B}\right|$. Then we have the following lemma.

Lemma 2. Let $\mathcal{D}$ be any derivation of $C$ from $I$ (we shall denote the thickness of $C$ with respect to a fixed derivation $\mathcal{D}$ as $\tau_{\mathcal{D}}$ ). Then there exists an ordered derivation $\mathcal{D}_{O}$ of $C$ from I with

$$
\tau_{\mathcal{D}}(C) \leq \tau_{\mathcal{D}_{O}}(C)
$$

Furthermore, if $\mathcal{D}_{1}$ and $\mathcal{D}_{2}$ are two ordered derivations of $C$ from $I$ then

$$
\tau_{\mathcal{D}_{1}}(C)=\tau_{\mathcal{D}_{2}}(C) .
$$

Proof. Proof of this can be found in [1] as the proof of Lemma 3.1.

We can see that the Cantor ternary set is ordered in the canonical construction and that this set has thickness 1 . In fact, for $0<a<1 / 2$, then $\tau\left(C_{a, a}\right)=\frac{a}{1-2 a}$.

\section{Results}

Now that our notation is in place we can relate thickness and the porosities. The derivation under consideration comes from the standard one where at stage $n$ (since only one interval is to be removed at a time in a derivation) we remove intervals in the order $O_{n, 1}, O_{n, 2}, \ldots, O_{n, 2^{n}}$. We will refer to this as the 
standard derivation. The first question we wish to look at concerns whether or not this derivation will be ordered. It seems obvious that the derivation will be ordered if the sequence of $a_{n}$ values is non-decreasing. However, this is not a necessary condition.

Lemma 3. The set $C_{a_{n}, a_{n}}$ with the standard derivation is ordered if

$$
a_{n+1} \geq \frac{3 a_{n}-1}{2 a_{n}} .
$$

Proof. Consider an interval $I$ from the $(n-1)$ st stage of the construction. The gap introduced in $I$ at the next stage will have length $\left(1-2 a_{n}\right)|I|$ and the gap length from the $(n+1)$ st stage will have length $\left(1-2 a_{n+1}\right) a_{n}|I|$. Setting $\left(1-2 a_{n+1}\right) a_{n}|I| \leq\left(1-2 a_{n}\right)|I|$ we arrive at our result.

This leads us to the immediate corollaries

Corollary 4. The set $C_{a_{n}, a_{n}}$ with the standard derivation is ordered if $a_{n} \leq$ $1 / 3$ for all $n$.

Proof. This comes from Lemma 4 since $a_{n} \leq 1 / 3$ implies $\frac{3 a_{n}-1}{2 a_{n}}$ is not positive.

So a Cantor set where $a_{2 n}=1 / 3$ and $a_{2 n+1}=1 / 5$ will be ordered even though the sequence of $a_{n}$ 's is not monotone. As a consequence of knowing our derivation is ordered we can easily find the thickness value for $C_{a_{n}, a_{n}}$.

Corollary 5. If $a_{n+1} \geq \frac{3 a_{n}-1}{2 a_{n}}$ then

$$
\tau\left(C_{a_{n}, a_{n}}\right)=\sup _{n \geq 1} \frac{a_{n}}{1-2 a_{n}} .
$$

Proof. Since Lemma 4 guarantees the standard derivation is ordered we get our result.

In finding a lower bound for the porosity index of the Cantor ternary set we looked at

$$
\frac{|O|}{\left|I^{j}\right|}
$$

where $O$ is the middle interval removed and $I^{j}$ is one of the intervals (left or right) resulting from removing $O$. In computing thickness, we used

$$
\frac{\left|I^{j}\right|}{|O|}
$$


The reciprocal nature of the fractions was the motivation behind our investigation of the relationship between the thickness $\tau(C)$ and the uniform index value $i(C)$.

Theorem 6. Let $C=C_{a_{n}, a_{n}}$ be a Cantor set constructed by the standard derivation. If $a_{n}$ is non-decreasing then

$$
i(C) \geq \frac{1}{\tau(C)} .
$$

Proof. Because $a_{n}$ is non-decreasing the standard derivation will be ordered. Hence $\tau(C) \geq \frac{a_{n}}{1-2 a_{n}}$ for each $n$, by Corollary 6 . On the other hand, at any stage the ratio of the length of the gap introduced in an interval $I$ versus the length of the new subinterval constructed is a lower bound for the values of the porosity index. This gives our result.

This brings us to the following corollary.

Corollary 7. Let $C=C_{a_{n}, a_{n}}$ be a Cantor set constructed by the standard derivation. If $a_{n}$ is non-decreasing then

$$
p(C) \geq \frac{1}{1+\tau(C)}
$$

Proof. This is simply an application of the relationship between the value of the porosity index at a point and the porosity at a point.

We have an example to show that it is necessary for $a_{n}$ to be non-decreasing. If we use $\left\{a_{n}\right\}$ with $a_{1}$ small and $a_{n}=1 / 3$ for $n \geq 2$ then the large gap in the first stage controls $\tau(C)$ while the $1 / 3$ 's control the porosity index. Therefore we can make the value of $\frac{1}{\tau(C)}$ exceed $i(C)$ (although the exact value for $i(C)$ is not known to this author, it is finite).

We note here that we do have similar results for non-centered Cantor sets. That is, $C_{a_{n}, b_{n}}$ where at the $n$th stage in the standard construction each of the $2^{n}$ intervals $I_{n, k}$ are divided into two subintervals $I_{n, k}^{0}$ and $I_{n, k}^{1}$ where $I_{n, k}^{0}$ and $I_{n, k}$ share the same left endpoint, $I_{n, k}^{1}$ and $I_{n, k}$ share the same right endpoint, and

$$
\frac{\left|I_{n, k}^{0}\right|}{\left|I_{n, k}\right|}=a_{n} \text { and } \frac{\left|I_{n, k}^{1}\right|}{\left|I_{n, k}\right|}=b_{n} .
$$

These results, however, are not as succinct as before. 
Lemma 8. The non-centered Cantor set $C_{a_{n}, b_{n}}$ is ordered in the standard derivation if

$$
\frac{1-a_{n}-b_{n}}{\max \left\{a_{n}, b_{n}\right\}} \geq 1-a_{n+1}-b_{n+1} .
$$

We can now give a result finding the thickness for the Cantor set $C_{a_{n}, b_{n}}$ assuming the $a_{n}$ and $b_{n}$ give an ordered derivation.

Lemma 9. If $\frac{1-a_{n}-b_{n}}{\max \left\{a_{n}, b_{n}\right\}} \geq 1-a_{n+1}-b_{n+1}$, then

$$
\tau\left(C_{a_{n}, b_{n}}\right)=\sup _{n}\left(\frac{\min \left\{a_{n}, b_{n}\right\}}{1-a_{n}-b_{n}}\right) .
$$

\section{Application}

The application we will look at is not truly new. It concerns the sum of Cantor sets. For two sets $A$ and $B$ we define their sum as

$$
A+B=\{a+b: a \in A \text { and } b \in B\}
$$

It is well-known that if $C$ is the Cantor ternary set then $C+C=[0,2]$. Our original intent was to find a strong connection between porosity of Cantor sets and the sum of these sets being an interval. Results about this are known concerning thickness. Theorem 10 comes from [4].

Theorem 10. For $j=1,2$ let $C_{j}$ be a Cantor set derived from $I_{j}$ with $O_{j} a$ gap of maximal size in $C_{j}$. Assume that

$$
\begin{aligned}
& \qquad\left|O_{1}\right| \leq\left|I_{2}\right| \text { and }\left|O_{2}\right| \leq\left|I_{1}\right| . \\
& \text { If } \tau\left(C_{1}\right) \cdot \tau\left(C_{2}\right) \geq 1 \text { then } C_{1}+C_{2}=I_{1}+I_{2} \text {. }
\end{aligned}
$$

In rephrasing this in terms of porosity and porosity index we need to put another restriction on our sets.

Corollary 11. For $j=1,2$ let $C_{j}=C_{a_{n}^{j}, a_{n}^{j}}$ be a Cantor set derived from $I_{j}$ with $O_{j}$ a gap of maximal size in $C_{j}$. Assume that the $a_{n}^{j}$ are non-decreasing and

$$
\left|O_{1}\right| \leq\left|I_{2}\right| \text { and }\left|O_{2}\right| \leq\left|I_{1}\right|
$$

If $i\left(C_{1}\right) \cdot i\left(C_{2}\right) \leq 1$ then $C_{1}+C_{2}=I_{1}+I_{2}$. 
Proof. Having $i\left(C_{1}\right) \cdot i\left(C_{2}\right) \leq 1$ is the same as

$$
1 \leq \frac{1}{i\left(C_{1}\right)} \cdot \frac{1}{i\left(C_{2}\right)} \leq \tau\left(C_{1}\right) \cdot \tau\left(C_{2}\right)
$$

and we apply Theorem 10 .

Corollary 12. For $j=1,2$ let $C_{j}=C_{a_{n}^{j}, a_{n}^{j}}$ be a Cantor set derived from $I_{j}$ with $O_{j}$ a gap of maximal size in $C_{j}$. Assume that the $a_{n}^{j}$ are non-decreasing for each $j$ and

$$
\left|O_{1}\right| \leq\left|I_{2}\right| \text { and }\left|O_{2}\right| \leq\left|I_{1}\right|
$$

If $p\left(C_{1}\right)+p\left(C_{2}\right) \leq 1$ then $C_{1}+C_{2}=I_{1}+I_{2}$.

ProOF. This is simply an application of Lemma 1.

Restating these results, if the "holes" in the Cantor sets are small enough, the sum of the sets is an interval. The relationship we used to get the corollaries was an inequality, however, not an equation. This leaves us with some room which means while the conditions are sufficient they are not necessary.

Example 1. Let $C$ be the Cantor ternary set. It is well-known that $p(C) \geq$ $3 / 4$. Thus $p(C)+p(C)>1$ while $C+C=[0,2]$.

So we have the following questions concerning porosities and sums of Cantor sets:

Questions How large can $p\left(C_{1}\right)+p\left(C_{2}\right)$ (or $\left.i\left(C_{1}\right) \cdot i\left(C_{2}\right)\right)$ be and still have $C_{1}+C_{2}$ be an interval? Is it possible for both to be strongly porous $(p(C)=1) ?$

Example 2. Theorem 5.11 in [3] leads us to an example of Cantor sets $C_{1}$ and $C_{2}$ which are strongly porous yet $C_{1}+C_{2}$ is an interval. We begin with the interval $[0,1]$. Let $C_{1}$ be the subset of $[0,1]$ with $x \in C_{1}$ if and only if $x$ has a zero in the $r$ th decimal place where $j !+1 \leq r \leq(j+1)$ ! where $j$ is odd. We construct $C_{2}$ in a similar manner except $j$ is even.

However, these sets do have thickness zero. So now we have a whole new set of questions.

Question If we have Cantor sets $C_{1}$ and $C_{2}$ so that $\tau\left(C_{1}\right) \cdot \tau\left(C_{2}\right) \geq 1$ how large can $p\left(C_{1}\right)+p\left(C_{2}\right)$ be? 
We have a partial answer to this, beginning with the constant Cantor set $C_{a, a}$. From the repeated removal of the same ratio from the middle we can calculate a lower bound of the porosity as

$$
p\left(C_{a, a}\right) \geq \frac{1-2 a}{(1-a)^{2}}
$$

(note that this is an improvement over our previous $1 / 2$ for the Cantor ternary set). We have already seen an exact value of $\tau\left(C_{a, a}\right)=\frac{a}{1-2 a}$ for the thickness. Then having both $C_{1}$ and $C_{2}$ be $C_{a, a}$ we have $\tau\left(C_{1}\right) \cdot \tau\left(C_{2}\right) \geq 1$ if $a \in$ $[1 / 3,1 / 2)$. Since porosity is a decreasing function of $a$ we have $p\left(C_{1}\right)+p\left(C_{2}\right)$ is bounded above by $p\left(C_{1 / 3,1 / 3}\right)+p\left(C_{1 / 3,1 / 3}\right)$ which is less than two (so an upper bound does exist for this question and this type of set).

We return to this result even if we do not insist $C_{1}$ and $C_{2}$ are the same constant Cantor set. When we have $C_{1}=C_{a, a}$ and $C_{2}=C_{b, b}$ the maximum of $p\left(C_{1}\right)+p\left(C_{2}\right)$ subject to $\tau\left(C_{1}\right) \cdot \tau\left(C_{2}\right) \geq 1$ will occur when $a=b=1 / 3$. So if the answer to the question is above $2 \cdot p\left(C_{1 / 3,1 / 3}\right)$, the Cantor sets involved must be non-constant.

Now Falconer's example tells us that adding the porosities of two Cantor sets cannot be used to indicate whether or not the sum of the sets is an interval. So we must ask if there is some type of indication we can get using porosity.

Question Does there exist an "elementary" operation $*$ defined on $[0,1]$ so that for Cantor sets $C_{1}$ and $C_{2}$

$$
p\left(C_{1}\right) * p\left(C_{2}\right) \leq 1
$$

if and only if $C_{1}+C_{2}$ is an interval?

\section{References}

[1] S. Astels, Cantor sets and numbers with restricted partial quotients, Trans. Amer. Math. Soc., 352(2000), no.1, 133-170.

[2] S. Astels, Thickness measures for Cantor sets, Elec. Research Announcements of the Amer. Math. Soc., 5(1999), 108-111.

[3] K. Falconer, The geometry of fractal sets, Cambridge University Press, 1985.

[4] S. Newhouse, The abundance of wild hyperbolic sets and non-smooth stable sets for diffeomorphisms, Inst. Hautes Études Sci. Publ. Math., 50(1979), 101-151. 
[5] J. Palis and F. Takens, Hyperbolicity and sensitive chaotic dynamics at homoclinic bifurcations, Cambridge University Press, Cambridge, 1993.

[6] B. Thomson, Real Functions, Lecture Notes in Math. (1170), SpringerVerlag, 1985.

[7] L. Zajíček, Porosity and $\sigma$-porosity, Real Anal. Exch., 13(1987-88), 314350. 